\title{
Retardation and facilitation of matching acquisition by differential outcomes
}

\author{
PETER J. URCUIOLI \\ Purdue University, West Lafayette, Indiana
}

\begin{abstract}
Pigeons were trained on many-to-one matching-to-sample with food and no-food outcomes that were either differential or nondifferential with respect to the sample stimuli. In the differential condition, outcomes were correlated with the correct comparison alternatives for half of the subjects, and were uncorrelated with those alternatives for the remaining subjects. Relative to nondifferential training, matching acquisition was facilitated in the correlated condition but retarded in the uncorrelated condition. These results clearly demonstrate that differential outcomes do not affect conditional discrimination learning merely by enhancing the discriminability or distinctiveness of the samples with which they are associated. Rather, they apparently give rise to another discriminative cue (viz., an outcome expectancy), which can either enhance or interfere with performance, depending on its predictive validity.
\end{abstract}

This paper is a report of an experiment that was designed to determine the mechanism(s) by which differential outcomes affect the acquisition of matching-to-sample (MTS) by pigeons. The effect itself is reasonably well established: acquisition is typically much faster when the reinforcer contingent upon correct choice differs as a function of the sample stimulus than when it does not (see, e.g., Edwards, Jagielo, Zentall, \& Hogan, 1982; Peterson, 1984; Santi \& Roberts, 1985b; Urcuioli, 1990b; Williams, Butler, \& Overmier, 1990). What is at issue is how this differential-outcomes effect (DOE) arises.

One explanation is that different outcomes make the sample stimuli with which they are associated more discriminable or distinctive than they would otherwise be (Colwill \& Rescorla, 1988; Edwards et al., 1982; Mackintosh, 1983; Peterson \& Trapold, 1982). Presumably, distinctive samples gain control over correct matching performances more readily than less distinctive ones do (Wright \& Sands, 1981; cf. Lawrence, 1949).

An alternative explanation appeals to the ability of subjects to anticipate trial outcomes prior to their matching choices (Honig \& Dodd, 1986; Peterson, 1984). According to this account, when outcomes differ as a function of the sample stimuli, those samples generate differentialoutcome expectancies which, in turn, provide an additional discriminative cue for performance (Honig, Matheson, \& Dodd, 1984; Peterson, 1984; Urcuioli, 1990b).

In a previous study that contrasted these two accounts (Urcuioli, 1990a), I trained two groups of pigeons on many-to-one MTS (cf. Santi \& Roberts, 1985a; Urcuioli,

This research was supported by NSF Grant 86-06926. The author is grateful to JeAndra Barner, Todd Hortman, Lisa Huston, Beth Kraemer, and Nicholas Ketterer for running the subjects. Reprint requests should be sent to the author, Department of Psychological Sciences, Purdue University, West Lafayette, IN 47907.
Table 1

A Schematic of the Correlated and Uncorrelated DiferentialOutcomes Conditions Run by Urcuioli (1990a), and Corresponding Theoretics Based Upon the Hypothesized Outcome Expectancies (Also Shown Is a Nondinerential Condition Not Included in the Urcuioli, 1990a, Design)

\begin{tabular}{lcl}
\hline \multicolumn{1}{c}{ Condition } & Matching Contingencies & Theoretics \\
\hline Correlated & $S_{1} \rightarrow C_{1}+(100 \%)$ & $S_{1} \cdot E_{100} \rightarrow C_{1}$ \\
(Differential) & $S_{2} \rightarrow C_{2}+(20 \%)$ & $S_{2} \cdot E_{20} \rightarrow C_{2}$ \\
& $S_{3} \rightarrow C_{1}+(100 \%)$ & $S_{3} \cdot E_{100} \rightarrow C_{1}$ \\
& $S_{4} \rightarrow C_{2}+(20 \%)$ & $S_{4} \cdot E_{20} \rightarrow C_{2}$ \\
Uncorrelated & $S_{1} \rightarrow C_{1}+(100 \%)$ & $S_{1} \cdot E_{100} \rightarrow C_{1}$ \\
(Differential) & $S_{2} \rightarrow C_{2}+(20 \%)$ & $S_{2} \cdot E_{20} \rightarrow C_{2}$ \\
& $S_{3} \rightarrow C_{1}+(20 \%)$ & $S_{3} \cdot E_{20} \rightarrow C_{1}$ \\
& $S_{4} \rightarrow C_{2}+(100 \%)$ & $S_{4} \cdot E_{100} \rightarrow C_{2}$ \\
Nondifferential & $S_{1} \rightarrow C_{1}+(60 \%)$ & $S_{1} \cdot E_{60} \rightarrow C_{1}$ \\
& $S_{2} \rightarrow C_{2}+(60 \%)$ & $S_{2} \cdot E_{60} \rightarrow C_{2}$ \\
& $S_{3} \rightarrow C_{1}+(60 \%)$ & $S_{3} \cdot E_{60} \rightarrow C_{1}$ \\
& $S_{4} \rightarrow C_{2}+(60 \%)$ & $S_{4} \cdot E_{60} \rightarrow C_{2}$ \\
\hline
\end{tabular}

Note- $S_{1}-S_{4}=$ four different sample stimuli. $C_{1}$ and $C_{2}=$ correct comparison alternatives associated with the samples. $E_{20}, E_{60}$, and $E_{100}=$ hypothesized expectancies associated with the $20 \%, 60 \%$, and $100 \%$ reinforcement schedules.

Zentall, Jackson-Smith, \& Steirn, 1989) with different probabilities of food reinforcement for correct choice. The matching contingencies for these groups are shown as the first two conditions in Table 1. For both groups, every correct choice was followed by food on trials beginning with two of four samples, whereas correct choices on the remaining trials were reinforced only $20 \%$ of the time. Since the samples were associated with different outcomes (viz., certain vs. uncertain food) for all birds, sample discriminability was equated across the two conditions. The groups differed from one another, however, in the relationship between the outcomes and correct choice. For the correlated group, each reinforcement probability was uniquely associated (correlated) with a particular correct comparison; for the uncorrelated group, the two probabil- 
ities were associated equally often with each comparison alternative. I found that MTS acquisition was faster, and subsequent working memory performances considerably more accurate, in the correlated than in the uncorrelated condition.

Clearly, these results cannot be explained in terms of sample distinctiveness. After all, if differential outcomes simply enhance the discriminability of the samples (and, hence, how quickly those stimuli acquire conditional stimulus control), then acquisition and retention by the correlated and uncorrelated groups in Urcuioli (1990a) should have been identical, not different. Instead, the results apparently reflect the differences in the "predictiveness" of the outcome expectancies associated with the sample stimuli. This hypothesized state of affairs is depicted in the Theoretics column of Table 1. For the correlated group, the expectation associated with the $100 \%$ reinforcement schedule $\left(\mathrm{E}_{100}\right)$ could act as a discriminative stimulus for choosing one comparison alternative $\left(\mathrm{C}_{1}\right)$, and the expectation associated with the $20 \%$ reinforcement schedule $\left(E_{20}\right)$ could act in a similar fashion for the other alternative $\left(\mathrm{C}_{2}\right)$. By contrast, those expectancies could not have served the same discriminative function in the uncorrelated group-in other words, they could not, by themselves, guide which choice those birds should make on any given trial. Thus, the Urcuioli (1990a) results provide good evidence in favor of the expectancy mediation account of the DOE (see also Honig et al., 1984)

Nonetheless, it is important to recognize that differential outcomes could alter sample discriminability and give rise to a potential expectancy cue. The Urcuioli (1990a) data simply show that the latter is sufficient to facilitate conditional discrimination learning by pigeons. But relative to a nondifferential condition (like that shown at the bottom of Table 1), samples associated with different outcomes might also be more distinctive. Detecting such a "discriminability" effect is difficult, however, when outcome expectancies can provide a redundant cue for choice, as in the Urcuioli (1990a) correlated condition. The problem is that it's impossible to disentangle sample-stimulus control from expectancy control merely by looking at performance during training. Consequently, measuring the former requires special test conditions in which the latter is effectively "removed."

In a separate study using the more typical two-sample, two-alternative procedure, Urcuioli (1990b) conducted just such an assessment after training birds on MTS with either differential or nondifferential outcomes. Contrary to the sample-discriminability account, however, he found that matching on the basis of the samples alone was no more accurate following differential training than it was following nondifferential training when the outcomes were different versus identical probabilities of food reinforcement, respectively. Furthermore, when the presence and absence of food reinforcement were the two outcomes, matching during the sample-only test was less accurate in the differential than in the nondifferential group. Thus, differential outcomes do not enhance sample distinctiveness when outcome expectancies provide another, valid cue for choice.

But what happens to sample distinctiveness when those expectancies are not predictive of choice, as in the uncorrelated group of Urcuioli (1990a)? Is sample-stimulus control in this situation potentiated relative to that in a nondifferential group? The answer here can presumably be obtained simply by comparing performances during training. Differentially trained birds should acquire MTS more quickly than nondifferentially trained controls, if the distinctiveness of the samples, which provide the only valid cue for choice in both groups, is enhanced for the former.

This prediction was tested in the present experiment. Pigeons learned many-to-one MTS with either differential or nondifferential outcomes. In one of the differential conditions, outcomes were correlated with correct comparison choice. In the other, they were uncorrelated with correct choice. The correlated group was predicted to show more rapid learning than the uncorrelated group, given previous findings that a valid expectancy cue will facilitate MTS acquisition (Honig et al., 1984; Urcuioli, 1990a). For the same reason, the correlated group was expected to learn faster than the nondifferential group. How acquisition would proceed in the uncorrelated versus nondifferential groups, however, was less certain. If differential outcomes enhance sample discriminability, the uncorrelated birds should acquire their matching task more rapidly than the nondifferential birds. On the other hand, if sample discriminability is unaffected by differential outcomes, their rates of acquisition should be similar.

For this experiment, the outcomes were the presence and absence of food. Since other data indicate that these events are particularly salient for pigeons (Urcuioli, 1990b), this should optimize the chances of detecting a sampledistinctiveness effect. Whether or not the Urcuioli (1990a) acquisition findings were replicable with other outcomes could also be assessed.

\section{METHOD}

\section{Subjects}

Twelve experimentally naive White Carneaux retired breeders obtained from Palmetto Pigeon Plant (Sumter, SC) participated in the experiment. All birds were initially reduced to $80 \%$ of their free-feeding body weights and were then maintained at that level by daily adjustment of the reinforcement duration in the experimental sessions. Supplemental feedings were given only when birds were unable to obtain enough food in each session to maintain an $80 \%$ weight, and on the 1 day per week that they were not run. All birds were housed individually in stainless steel, wire cages in a colony room with a 14:10-h light:dark cycle. Water and grit were freely available at all times in the home cage. Just prior to the start of the experiment, the birds were grouped into triplets on the basis of similar body weights. They were then randomly assigned to one of the three groups described below. 


\section{Apparatus}

A single BRS/LVE chamber (Model SEC-002) equipped with a three-key intelligence panel (Model PIP-016) was used for the experiment. The pigeon's compartment and the layout of the panel have been described in detail elsewhere (e.g., in Urcuioli, 1990b). Briefly, stimuli were displayed on the three horizontally aligned, circular pecking keys by means of back-mounted inline projectors (Model IC-901-IDD). Center-key stimuli were three white vertical (V) and horizontal (H) lines on a black background, and white (W), blue (B), and yellow (Y) homogeneous fields (Pattern No. 692). The side-key stimuli were red (R) and green (G) fields. General chamber illumination was provided by a partially covered GE No. 1829 bulb mounted above the center key, which directed light toward the ceiling. A $5 \times 5.8 \mathrm{~cm}$ opening for the food magazine was centered about $13 \mathrm{~cm}$ below the center key. The apparatus was continually ventilated by a blower fan attached to the exterior of the chamber. The sound of the fan also helped to mask extraneous noises. An IBM PC/XT located in an adjacent room programmed and recorded all experimental events.

\section{Procedure}

Preliminary training for all birds consisted of initial training to eat from the food hopper, followed by training to peck each of the three keys when it was illuminated by one of the stimuli indicated above. Keypecking to a $\mathbf{W}$ stimulus on the center key was initially established via the method of successive approximations. The birds were then allowed to collect $\mathbf{6 0}$ food reinforcers for pecking this stimulus. On subsequent days, the birds received 30 reinforcers each for pecking $V$ and $H$ center-key lines, and $B$ and $Y$ center-key hues. On the final preliminary training session, both the center and side keys were lit on successive trials with the stimuli that birds would subsequently encounter during MTS. Red and green hues appeared 10 times on the left and right side keys, and V, H, B, and Y appeared 10 times on the center key, with a single peck to each stimulus producing 3-sec access to grain. Successive trials were separated by a 10-sec intertrial interval (ITI), and the houselight was on throughout each session.

Next, birds received nine sessions of "pretraining" with the stimuli that would later serve as samples in MTS. The first three sessions involved only $V$ and $H$ lines, the next three only the $B$ and $Y$ hues, and the last three all four stimuli. Each session consisted of 60 trials divided equally among the stimuli scheduled to appear in that session. Trials began with presentation of a W "ready" stimulus on the center key. A single peck to W immediately turned it off and produced the trial stimulus on the same key $500 \mathrm{msec}$ later. That stimulus remained on for a fixed duration of $3 \mathrm{sec}$, after which it was turned off and followed by either food reinforcement or by presentation of the food-hopper light only (no food). The stimulus-outcome relationships experienced by each bird depended on its group assignment (see below). Successive pretraining trials were separated by a 10 -sec ITI, the first 9 sec of which were spent in darkness. The houselight then came on for the last $1 \mathrm{sec}$ of the ITI (i.e., $1 \mathrm{sec}$ prior to the W "ready" stimulus), and it remained on until the end of the reinforcement cycle or the equivalent no-food period. The duration of food reinforcement varied from 2 to $6 \mathrm{sec}$ across sessions for individual birds in a manner that maintained their body weights as close to the $80 \%$ level as possible.

For the 4 birds assigned to Group Nondiff, food and no food occurred about equally often following each center-key stimulus. Food was presented when the output of the computer's random number generator at stimulus offset was less than or equal to .5; otherwise, no food was presented. For the 4 birds assigned to Group Corr and the 4 assigned to Group Uncorr, food always occurred following one of the two line orientations, and one of the two hues. The remaining stimuli always ended with no food. The stimulus-outcome relationships were counterbalanced within each group with the constraint that, for each Corr-Uncorr pair of birds within a triplet, the assignments were identical with respect to the two line orientations (see Table 2 below).

Sixty sessions of acquisition on many-to-one MTS followed the completion of pretraining. The 96 trials of each MTS session were equally divided among the four sample stimuli ( $\mathrm{V}, \mathrm{H}, \mathrm{B}$, and $\mathrm{Y}$ ). Each trial began with the center-key presentation of a $W$ "ready" stimulus. A single peck to $\mathrm{W}$ immediately turned it off and produced the sample for that trial $500 \mathrm{msec}$ later. The sample remained on for $3 \mathrm{sec}$, independently of the bird's responses, after which time it was turned off and immediately followed by $R$ and $G$ comparison hues on the side keys. For all birds, the $\mathbf{R}$ comparison was "correct" on V-and B-sample trials, and G was "correct" on $\mathrm{H}$ - and Y-sample trials.

As is shown in Table 2, the three groups differed in the outcomes associated with the correct choice responses. For Group Nondiff, a single peck to the correct comparison alternative produced the food and no-food outcomes with equal probability following each sample. For the remaining two groups, the outcomes were differential with respect to the samples. In Group Corr, 2 birds received food after every correct choice on V-and B-sample trials, and no food after every correct choice on $\mathrm{H}$ - and $\mathrm{Y}$-sample trials. The other 2 subjects had the opposite assignments. For all Group Corr birds, however, food and no food were correlated with the correct comparisons. By contrast, food and no food were uncorrelated with those alternatives in Group Uncorr. For these subjects, one outcome occurred after every correct choice on $\mathrm{V}$ - and $\mathrm{Y}$-sample trials, and the other after every correct choice on $\mathrm{H}$ - and $\mathrm{B}$-sample trials, with sample-outcome relationships counterbalanced across subjects.

In all three groups, pecking the incorrect comparison alternative on any trial immediately turned the houselight off for a period equal to the reinforcement duration. Then, following the ITI, the trial was repeated until the bird made the correct choice (i.e., a correction procedure was used). Successive matching trials were separated by a 10-sec ITI, the first 9 sec of which were spent in darkness. The houselight was turned on for the last $1 \mathrm{sec}$ of the ITI, and it remained on until the end of the food or no-food period following a correct choice, or until an incorrect choice was made. Reinforcement duration was again adjusted on a session-by-session basis for each bird in a manner that maintained its body weight around the $80 \%$ level.

The experiment was run in two replications. Since the data from the replications were comparable, they have been collapsed for

Table 2

Experimental Design

\begin{tabular}{|c|c|}
\hline Group & Matching Contingencies \\
\hline Corr & $\begin{array}{l}V \cdot \text { FT } 3 \mathrm{sec} \rightarrow \mathrm{R}+(\text { (food) } \\
\mathrm{H} \cdot \text { FT } 3 \mathrm{sec} \rightarrow \mathrm{G}+\text { (no food) } \\
\text { B.FT } 3 \mathrm{sec} \rightarrow \mathrm{R}+(\text { food) } \\
\mathrm{Y} \cdot \text { FT } 3 \mathrm{sec} \rightarrow \mathrm{G}+(\text { no food })\end{array}$ \\
\hline Uncorr & $\begin{array}{l}\text { V } \cdot \text { FT } 3 \mathrm{sec} \rightarrow \mathrm{R}+(\text { food) } \\
\mathrm{H} \cdot \text { FT } 3 \mathrm{sec} \rightarrow \mathrm{G}+\text { (no food) } \\
\text { B. FT } 3 \mathrm{sec} \rightarrow \mathrm{R}+(\text { no food) } \\
\mathrm{Y} \cdot \text { FT } 3 \mathrm{sec} \rightarrow \mathrm{G}+(\text { food })\end{array}$ \\
\hline Nondiff & $\begin{array}{l}V \cdot \text { FT } 3 \mathrm{sec} \rightarrow \mathrm{R}+(\text { food/no food }) \\
\mathrm{H} \cdot \text { FT } 3 \mathrm{sec} \rightarrow \mathrm{G}+(\text { food/no food) } \\
\text { B } \text {. FT } 3 \mathrm{sec} \rightarrow \mathrm{R}+(\text { food/no food }) \\
\mathrm{Y} \cdot \text { FT } 3 \mathrm{sec} \rightarrow \mathrm{G}+(\text { food/no food })\end{array}$ \\
\hline
\end{tabular}

Note $-V=$ three white vertical lines on a black background, $H=$ three white horizontal lines on a black background, $\mathrm{B}=$ blue homogeneous field, $\mathbf{Y}=$ yellow homogeneous field, $\mathbf{R}=$ red homogeneous field, $\mathbf{G}=$ green homogeneous field. Samples appear to the left of the arrows, and correct comparisons appear to the right. Outcomes following correct choice are shown inside the parentheses. Incorrect comparisons and counterbalancing of sample-outcome relationships in Groups Corr and Uncorr are omitted for clarity. 
presentation and analysis. The primary dependent variable, the percentage of correct choice responses, was computed on the basis of the first choice on each trial. In other words, correct responses following incorrect choices did not enter into the computation. Statistical significance in the analyses of the results was judged relative to the tabled $F$ values provided by Rodger (1975), using a Type I error rate of .05 .

\section{RESULTS}

Figures 1 and 2 show MTS acquisition over blocks of two sessions for the subjects in each triplet. In all cases, the Group Corr birds showed the most rapid acquisition of many-to-one matching, the Group Uncorr birds the slowest, and the Group Nondiff birds a rate in between these two extremes. An analysis of variance (ANOVA) on the acquisition data showed a significant overall group effect $[F(2,9)=24.96]$, a significant overall effect of training session $[F(29,261)=66.16]$, and a significant group $\times$ session interaction $[F(58,261)=7.22]$. The mean levels of accuracy averaged over all sessions were 96.1, 83.4, and 72.2\% correct for Group Corr, Nondiff, and Uncorr, respectively. An ANOVA on these means confirmed that (1) overall accuracy was higher in Group Corr than in Group Uncorr $[F(2,9)=24.94]$, and (2) accuracy of performance by Group Nondiff was no different than the average of the other two groups $[F(2,9)=.03]$, thus indicating that it was intermediate between that of Groups Corr and Uncorr.

The difference between Group Corr and Group Uncorr replicates the previous findings of Urcuioli (1990a), and it is consistent with an interpretation in terms of outcome expectancy mediation. So too is the difference between Group Corr and Group Nondiff. More importantly, how-
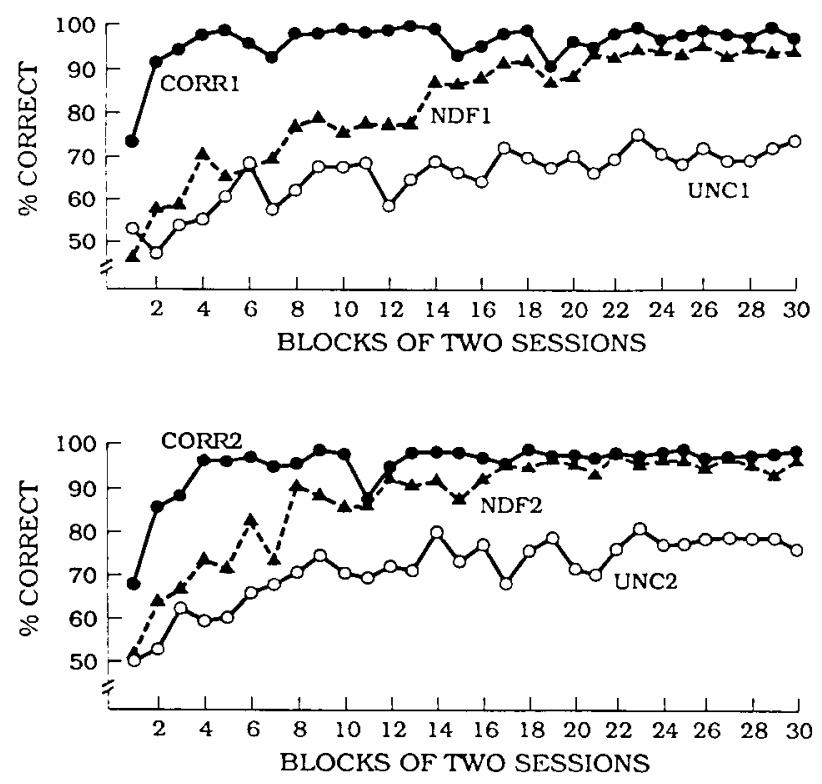

Figure 1. Acquisition of many-to-one matching-to-sample over blocks of two sessions for two of the four triplets of subjects.
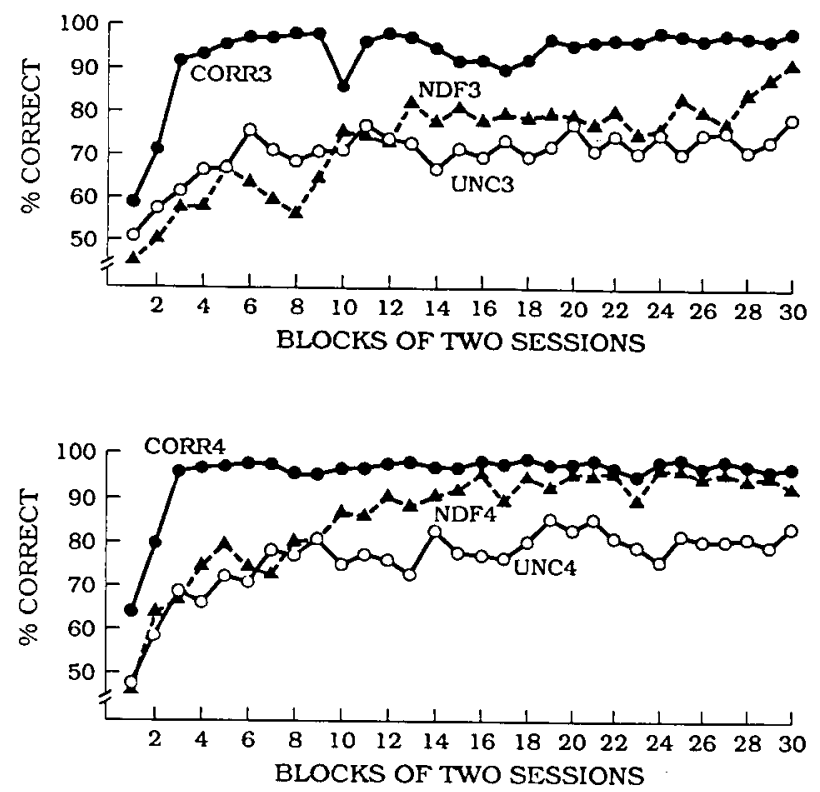

Figure 2. Acquisition of many-to-one matching-to-sample over blocks of two sessions for the remaining two triplets of subjects.

ever, the finding that Group Uncorr did not learn to match more quickly than Group Nondiff indicates that differential outcomes did not enhance sample distinctiveness even when outcome expectancies were not predictive of choice (cf. Honig et al., 1984). But the acquisition data from the latter two groups are not just inconsistent with the samplediscriminability hypothesis; they are the exact opposite of what that hypothesis would predict. The fact that Group Uncorr learned more slowly than Group Nondiff indicates that the different outcomes in the former group appeared to have interfered with sample-stimulus control. Indeed, even after 60 sessions of training (5,760 MTS trials), the Group Uncorr birds were matching at only $79.4 \%$ accuracy (compared to $99.2 \%$ in Group Corr and $95.6 \%$ in Group Nondiff). Why did differential outcomes produce such a disruptive effect?

One possibility is that Group Uncorr's poor performance reflects the conflicting information provided by their differential-outcome expectancies. For example, in the presence of a food expectancy, to choose the R comparison was correct on some trials, but to choose $G$ was correct on others. The same held for the no-food expectancy. Consequently, correct performance on one trial may have adversely affected matching performance on the subsequent trial. In particular, this view predicts that on two successive trials involving the same expectancy, matching accuracy would be lower when the samples giving rise to that expectancy differed across trials than when they remained the same. To evaluate the possible impact of such sequential effects, accuracy on every trial involving the expectation of food was computed as a function of whether the food-associated sample for that trial (trial $n$ ) was the same as or different from the food- 
associated sample from the preceding trial (trial $n-1$ ). Analogous computations were also made for successive trials involving the expectation of no food. Table 3 shows the results of these analyses for each Group Uncorr bird, averaged over the last 30 acquisition sessions. Contrary to the predictions above, matching accuracy was not significantly reduced when trial $n$ involved the alternative sample associated with the same expectancy as that for the sample on trial $n-1\left[F_{\mathrm{s}}(1,3)=2.30\right.$ and 0.11 for the food and no-food expectancies, respectively].

Another possibility is that the Group Uncorr birds simply tended to repeat food-reinforced responses from immediately preceding trials, and to switch to the alternative comparison when the prior correct choice yielded no food. (Note that since a correction procedure was used, the immediately prior choice was always "correct.") If these birds did perseverate in pecking the same comparison that had just yielded food reinforcement, matching accuracy should have been lower when the current correct choice differed from that on trial $n-1$ than when it was the same as the food-reinforced choice on trial $n-1$. The opposite pattern of results would be expected when the immediately preceding choice produced the no-food outcome: matching accuracy should have been lower when the correct choice on trial $n$ was the same as the immediately prior choice than when it was different. Table 4 presents the results of these sequential-effects analyses. Here, the overall pattern of results runs counter to what food-reinforced perseveration, and no-food-reinforced switching, would predict. For example, the top portion of Table 4 shows that the Group Uncorr birds were less likely to select the same comparison as the one producing food on the prior trial than they were to peck the alternative comparison $[F(1,3)=22.78]$. Conversely, the bottom portion of the table shows that they were more likely to select the same comparison as the one produc-

Table 3

Matching Accuracy for the Group Uncorr Birds Over the Last 30 Acquisition Sessions as a Function of Whether the Same Expectancy on Two Successive Trials was Produced by the Same or Different Samples

\begin{tabular}{ccc}
\hline & Same & Different \\
Bird & Sample & Sample \\
\hline
\end{tabular}

Percent correct choice on food-expectancy trial $n$ vis-à-vis the foodexpectancy sample on trial $n-1$ :

$\begin{array}{lll}\text { UNC1 } & 91.8 & 92.3 \\ \text { UNC2 } & 97.9 & 97.2 \\ \text { UNC3 } & 97.4 & 94.7 \\ \text { UNC4 } & 98.1 & 97.0 \\ M & 96.3 & 95.3\end{array}$

Percent correct on no-food-expectancy trial $n$ vis-à-vis the no-foodexpectancy sample on trial $n-1$ :

$\begin{array}{lll}\text { UNC1 } & 44.6 & 50.6 \\ \text { UNC2 } & 58.8 & 61.1 \\ \text { UNC3 } & 57.5 & 48.0 \\ \text { UNC4 } & 70.1 & 66.7 \\ M & 57.8 & 56.6\end{array}$

Table 4

Erfects of Immediately Prior Choices on Performance by the Group Uncorr Birds Over the Last 30 Acquisition Sessions

\begin{tabular}{ccc} 
Bird & $\begin{array}{c}\text { Same } \\
\text { Choice }\end{array}$ & $\begin{array}{c}\text { Different } \\
\text { Choice }\end{array}$ \\
\hline
\end{tabular}

Percent correct choices on trial $n$ vis-à-vis the food-reinforced choice on trial $n-1$ :

$\begin{array}{lll}\text { UNC1 } & 62.2 & 74.1 \\ \text { UNC2 } & 69.6 & 77.2 \\ \text { UNC3 } & 69.2 & 73.2 \\ \text { UNC4 } & 76.8 & 84.2 \\ M & 69.4 & 77.2\end{array}$

Pencent correct choices on trial $n$ vis-à-vis the no-food correct choice on trial $n-1$ :

\begin{tabular}{lll} 
UNC1 & 76.9 & 70.1 \\
UNC2 & 84.0 & 76.1 \\
UNC3 & 83.5 & 69.2 \\
UNC4 & 89.6 & 80.9 \\
$M$ & 83.5 & 74.1 \\
\hline
\end{tabular}

ing no food on the prior trial than they were to peck the alternative comparison $[F(1,3)=31.81]$.

Another explanation to consider is that the inconsistent relationship between outcomes and correct choice actually interfered with the ability of the Group Uncorr birds to accurately discriminate between the samples. Perhaps they were more likely to confuse $\mathrm{V}$ with $\mathrm{H}$, and $\mathrm{B}$ with $Y$, than the other birds. One way to evaluate this sampleconfusion hypothesis is to examine how the birds responded to the samples themselves. With little confusion (i.e., good sample discrimination), birds should respond quickly and rapidly to the food-associated samples, but seldom to the no-food-associated samples. Nondiscriminative sample behavior would indicate sample confusion. Table 5 shows the average sample-response rates and the average sample-response latencies for the Group Uncorr birds over the last 30 training sessions. Also shown for comparison purposes are the corresponding data from Group Corr. ${ }^{1}$ Clearly, the birds in Group Uncorr showed every bit as good a discrimination between the samples as did the birds in Group Corr (perhaps even better). They responded quickly and frequently to the samples associated with food, and slowly and infrequently to the samples associated with no food. Thus, matching performances by the Group Uncorr birds are not explicable in terms of greater confusion between the samples. To the contrary, the data indicate that these birds knew exactly what outcome was scheduled for the end of each trial.

With respect to this finding, were matching performances in Group Uncorr selectively depressed on trials involving no food, or were they generally depressed on all trials? In Figures 3 and 4, accuracy on the food and no-food trials is plotted for each Uncorr bird during acquisition and, for comparison purposes, for each Corr bird. These figures show very clearly that the poor overall level of performance exhibited by the Uncorr birds during acquisition was due almost entirely to their very low levels of accuracy on no-food trials. For 3 of the 4 
Table 5

Sample Response Rates and Sample Response Latencies for Group Uncorr and Group Corr Over the Last 30 Training Sessions

$\begin{array}{lcc}\text { Sample-Outcome } & \begin{array}{c}\text { Group Uncorr } \\ \text { Response Rate }\end{array} & \text { Group Corr } \\ \text { Line-food } & 2.21 & \\ \text { Line-no food } & .18 & 2.22 \\ \text { Hue-food } & 2.08 & .25 \\ \text { Hue-no food } & .21 & 1.38 \\ & \text { Latency } & .31 \\ \text { Line-food } & .25 & \\ \text { Line-no food } & 2.28 & .80 \\ \text { Hue-food } & .22 & 1.66 \\ \text { Hue-no food } & 1.98 & 1.02 \\ \end{array}$

Note--Response rate is given in responses/second; latency is in seconds.

birds in this group, there was essentially no improvement in accuracy on these trials from the 1st to the 60th acquisition session. By contrast, matching performance on food trials improved rapidly with training and stabilized at a level close to that exhibited by Group Corr. For the Group Corr birds, matching on no-food trials was acquired more slowly than on food trials, but nonetheless quite rapidly: $90 \%$ accuracy was achieved within 6-8 sessions. Indeed, acquisition on the no-food trials in Group Corr was as rapid as on the food trials in Group Uncorr.

\section{DISCUSSION}

The major finding from this experiment is that differential outcomes facilitated MTS acquisition only to the extent that those outcomes were correlated with the correct comparison alternatives. When different outcomes were associated with the sample stimuli but those outcomes were uncorrelated with the comparison alternatives, pigeons were noticeably slower in acquiring accurate matching than they were when each outcome occurred equally often on all trials. These results are yet another indication that the DOE arises when subjects' differentialoutcome expectancies provide a reliable cue for later performance. More importantly, the data effectively rule out enhanced sample discriminability as a viable, alternative explanation. That account anticipates faster matching acquisition with differential outcomes (as opposed to nondifferential outcomes) independently of their correlation with correct choice. The finding that Group Uncorr acquired more slowly than Group Nondiff disconfirms this prediction (cf. Peterson \& Trapold, 1982).

The latter result is intriguing as well as theoretically discriminating. For example, the samples in Group Uncorr provided just as valid a cue for correct choice as they did in Group Nondiff, yet they supported a much lower level of accuracy. Apparently, the Group Uncorr birds were unable to disregard the different end-of-trial outcomes associated with their samples, and this interfered with sample-stimulus control. The locus of the interference was identified, but its "source" remains unclear. Specifically, the low overall level of matching accuracy resulted from selectively poor performance on trials in which correct choices ended in no food. At first glance, this is quite understandable, given that a lower valued outcome was being anticipated. In other words, why choose the correct comparison when that choice will not produce food?

There are two problems with this account. First, choice of the correct comparison advanced the bird to the next, and possibly food-reinforced, trial. Second, and more importantly, the same line of reasoning applies to Group Corr, yet that group did not show the same effect. Two of their four samples also predicted no food, but accuracy on these trials quickly approached the level seen with the food outcome. The problem, then, is to explain why nofood performances differed so dramatically in the two differential-outcomes groups.

One possibility might be that sample-outcome associations were somewhat stronger in Group Uncorr than in Group Corr and that this difference led to a weaker association between samples and correct comparisons in Group Uncorr. One reason for supposing that sampleoutcome associations might have been stronger in Group Uncorr is that the food and no-food outcomes were associated only with the samples in this group, whereas in Group Corr they were associated with the R and G correct comparisons as well. But even if sample-outcome associations differed in strength between groups, the idea that they would more effectively "block" the development of conditional stimulus control by the samples in
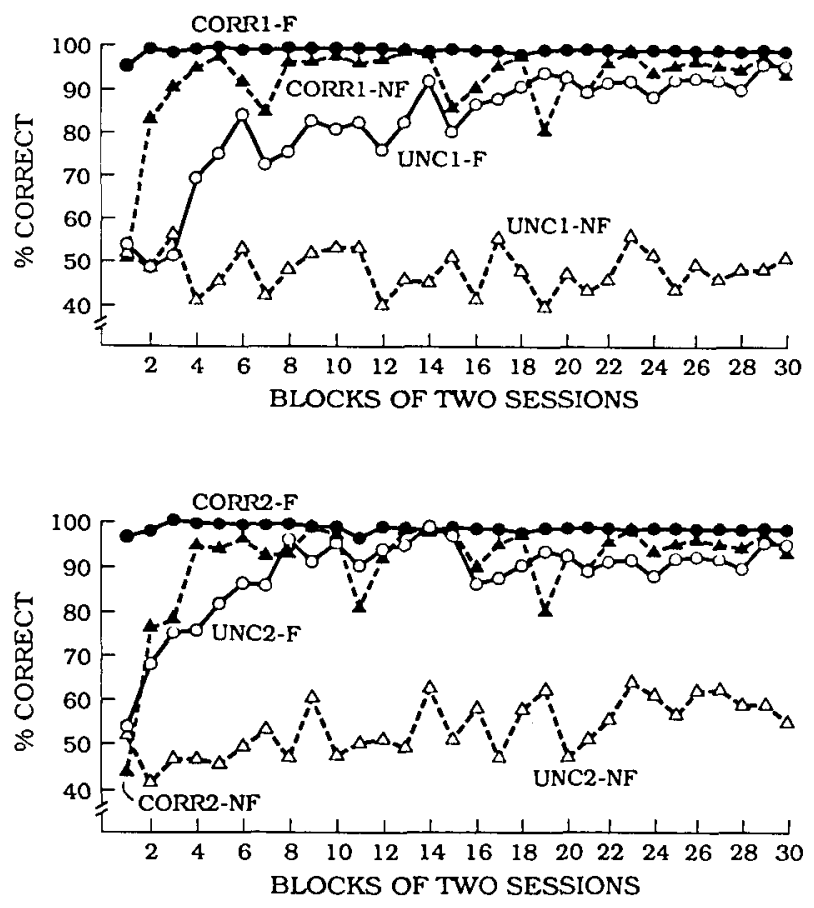

Figure 3. Percentage of correct choice responses on trials with samples associated with food (F) and no food (NF) for two of the four pairs of Corr and Uncorr birds during acquisition. 

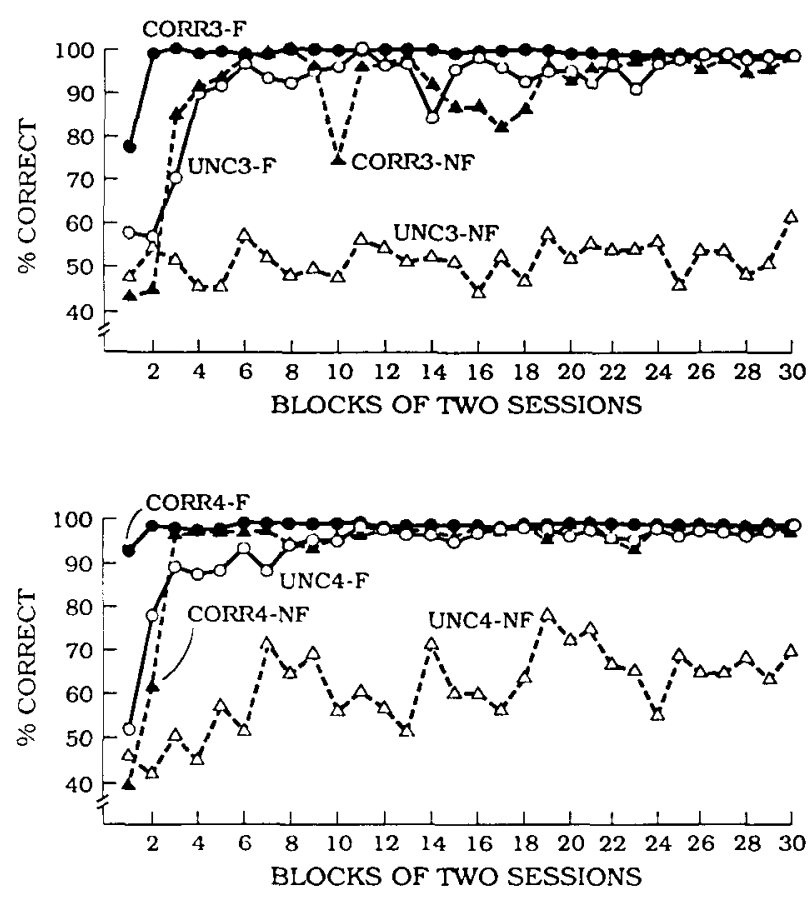

Figure 4. Percentage of correct choice responses on trials with samples associated with food (F) and no food (NF) for the remaining two pairs of Corr and Uncorr birds during acquisition.

Group Uncorr is unsatisfactory. For one, it doesn't explain the difference in conditional stimulus control on food and no-food trials in Group Uncorr. In other words, if the sample-outcome associations interfere with samplecorrect comparison associations, then why shouldn't this interference extend equally to all trials? Second, previous data (Urcuioli, 1990b) indicate that sample-correct comparison associations in MTS with food and no-food outcomes are relatively weak when the expectation of those outcomes provides a predictive cue for choice, as in Group Corr of the present study. By implication, then, conditional stimulus control by the samples should be weaker in Group Corr, not in Group Uncorr. Unfortunately, it was impossible to conduct an independent and meaningful sample-only test (Urcuioli, 1990b) to evaluate this in the present experiment, because the uncorrelated birds never learned to match at levels of accuracy comparable to those of their correlated counterparts (viz., above $90 \%$ correct).

Another account (Thomas Zentall, personal communication) focuses on the possible effects of differences in comparison-response biases across the groups. For Group Corr, one comparison stimulus was always followed by food when correct, and the other was always followed by no food. Consequently, any strong preference to peck the food-associated alternative early in acquisition would likely cause these birds to select the incorrect alternative repeatedly on no-food trials. Frequent repeat errors might then enhance their attention to the relevant trial characteristics/relationships, hence hastening acquisition.
By contrast, since there was no reason for a similar comparison preference to develop in Group Uncorr (because each correct comparison was followed equally often by food), these birds may have switched sooner to the alternative comparison following an incorrect choice. This "rapid switching" could have slowed the development of conditional stimulus control by the samples, especially on trials in which birds knew that food was not forthcoming. For example, if the uncorrelated birds adopted a strategy of, say, randomly pecking either comparison or either side key on no-food trials and then switching immediately to the other comparison or to the other side key if incorrect, they would not have effectively learned the sample-correct comparison associations on these trials. This attentional account thus requires fewer repeat errors in Group Uncorr than in Group Corr, but just the opposite was obtained. For instance, over the first 10 acquisition sessions, the 4 Group Uncorr birds made a total of 1,839 repeat errors, compared to 1,000 for the Group Corr birds.

Despite the lack of resolution of this particular issue, the overall pattern of results is nonetheless informative with regard to the general explanation of the DOE described earlier and to others as well. For example, Colwill and Rescorla (1988, p. 162) have recently suggested that differences in comparison-outcome associations could be responsible for the different rates of conditional discrimination learning typically observed in differentialoutcomes studies. After all, when different outcomes are associated with the samples, those outcomes typically have a unique association with the correct comparisons as well. This "comparison-distinctiveness" explanation of the DOE would certainly predict that Group Corr should learn faster than both Group Nondiff and Group Uncorr.

However, it cannot explain why performance in the latter two groups should differ. Since the R and G comparisons in both of those groups had the same, nondifferentialoutcome associations overall, similar rates of acquisition would be anticipated. The fact that Group Uncorr acquired more slowly than Group Nondiff indicates that something more than comparison-outcome associations was involved. The most likely candidate is the nature of the sample-outcome associations, and the expectancies to which they give rise. When expectancies differed in discriminable ways across trials but were not predictive of correct choice, they interfered with accurate matching performance relative to nondifferential expectancies. Whether or not this interference effect is a general one (i.e., can it be obtained with other types of outcomes?) and exactly how it is produced are some issues for future research.

\section{REFERENCES}

Colwill, R. M., \& Rescorla, R. A. (1988). Associations between the discriminative stimulus and the reinforcer in instrumental learning. Journal of Experimental Psychology: Animal Behavior Processes, 14, 155-164

Edwards, C. A., Jagielo, J. A., Zentall, T. R., * Hogan, D. E. (1982). Acquired equivalence and distinctiveness in delayed matching to sample by pigeons: Mediation by reinforcer-specific expectan- 
cies. Joumal of Experimental Psychology: Animal Behavior Processes, 8, 244-259.

HoNIG, W. K., \& DoDD, P. W. D. (1986). Anticipation and intention in working memory. In D. F. Kendrick, M. E. Rilling, \& M. R. Denny (Eds.), Theories of animal memory (pp. 77-100). Hillsdale, NJ: Erlbaum.

Honig, W. K., Matheson, W. R., \& Dodd, P. W. D. (1984), Outcome expectancies as mediators for discriminative responding. Canadian Journal of Psychology, 38, 196-217.

LAWRENCE, D. H. (1949). Acquired distinctiveness of cues: I. Transfer between discriminations on the basis of familiarity with the stimulus. Journal of Experimental Psychology, 39, 770-784.

Mackintosh, N. J. (1983). Conditioning and associative learning. New York: Oxford University Press.

Peterson, G. B. (1984). How expectancies guide behavior. In H. L. Roitblat, T. G. Bever, \& H. S. Terrace (Eds.), Animal cognition (pp. 135-148). Hillsdale, NJ: Erlbaum.

Peterson, G. B., \& Trapold, M. A. (1982). Expectancy mediation of concurrent conditional discriminations. American Journal of Psychology, 95, 571-580.

RoDGER, R. S. (1975). The number of non-zero, post hoc contrasts from ANOVA and error rate: I. British Journal of Mathematical \& Statistical Psychology, 28, 71-78.

SANTI, A., Roberts, W. A. (1985a). Prospective representation: The effects of varied mapping of sample stimuli to comparison stimuli and differential trial outcomes on pigeons' working memory. Animal Learning \& Behavior, 13, 103-108.

SANTI, A., \& RoBeRTS, W. A. (1985b). Reinforcement expectancy and trial spacing effects in delayed matching-to-sample by pigeons. Animal Learning \& Behavior, 13, 274-284.
UrCuIOLI, P. J. (1990a). Differential outcomes and many-to-one matching: Effects of correlation with correct choice. Animal Learning \& Behavior, 18, 410-422.

URCUIOL, P. J. (1990b). Some relationships between outcome expectancies and sample stimuli in pigeons' delayed matching. Animal Leaming \& Behavior, 18, 302-314.

Urcuioli, P. J., Zentall, T. R., Jackson-Smith, P., \& Steirn, J. N. (1989). Evidence for common coding in many-to-one matching: Retention, intertrial interference, and transfer. Joumal of Experimental Psychology: Animal Behavior Processes, 15, 264-273.

Williams, D. A., Butler, M. M., \& Overmier, J. B. (1990). Expectancies of reinforcer location and quality as cues for a conditional discrimination in pigeons. Joumal of Experimental Psychology: Animal Behavior Processes, 16, 3-13.

Wright, A. A., \& SANDS, S. F. (1981). A model of detection and decision processes during matching-to-sample by pigeons: Performance with 88 different wavelengths in delayed and simultaneous matching tasks. Journal of Experimental Psychology: Animal Behavior Processes, 7, 191-216.

\section{NOTE}

1. In Group Nondiff, response rates and latencies were approximately equal for the two line samples and for the two hue samples. Average rates for $\mathrm{V}, \mathrm{H}, \mathrm{B}$ and $\mathrm{Y}$ were $2.45,2.24,2.04$, and 1.94 responses $/ \mathrm{sec}$, respectively. The corresponding latencies were $.23, .24, .44$, and $.62 \mathrm{sec}$, respectively.

(Manuscript received June 25, 1990;

revision accepted for publication September 27, 1990.) 